INVITED ARTICLE

\title{
Thrombocytopenia in Intensive Care Unit
}

\author{
Narmada Aluru ${ }^{1}$, Srinivas Samavedam ${ }^{2}$ \\ Indian Journal of Critical Care Medicine (2019): 10.5005/jp-journals-10071-23249
}

\section{INTRODUCTION}

Thrombocytopenia is one of the most common hematological abnormalities encountered in the ICU, with an incidence ranging from 13 to $65 \%$. Incidence varies between different ICUs and across different groups of patient population. Incidence is highest in adult and medical ICUs compared to pediatric and surgical units. ${ }^{1,2}$ Thrombocytopenia is defined as an absolute platelet count $<150,000 / \mu \mathrm{L}$ or a decrement greater than $50 \%$ from baseline value. The threshold for defining thrombocytopenia is varied among different studies, with some studies defining it as a count $<100,000$ / $\mu \mathrm{L}$, and this is the reason for wide variation in the incidence. ${ }^{2}$

\section{Mechanisms}

Platelets are versatile cells with a wide range of functions, taking part in several disease affecting different organs and organ systems. Normally, a complex interplay between the bone marrow, liver and the RES, maintains a constant platelet count in an individual. In critically ill patients mechanisms contributing to thrombocytopenia are poorly understood. The reasons for thrombocytopenia in ICU are numerous and often multiple mechanisms act simultaneously, ${ }^{2}$ but can in general be divided into central causes (impaired production and/or dysfunction of the bone marrow) and peripheral causes (platelet destruction, increased sequestration, or pharmaceutically induced thrombocytopenia, hemodilution). The common mechanisms are (Table 1): ${ }^{3}$

- Decreased production

- Increased sequestration of platelets

- Increased destruction

Table 1: Mechanisms of thrombocytopenia

\begin{tabular}{ll}
\hline Mechanism & Example \\
\hline Increased consumption & - DIC, TTP, HELLP, PTE \\
Decreased production & - Chemotherapy \\
& - Radiotherapy \\
& - Sepsis, viral infections \\
Increased sequestration & - Hypersplenism \\
& - CHF \\
Increased destruction & - Hypothermia \\
& Nonimmune: TTP, DIC, sepsis, \\
& drugs, mechanical devices \\
Hemodilution & Immune: HIT, ITP \\
& Drugs, sepsis \\
Pseudothrombocytopenia & - Transfusion of fluids \\
& - EDTA-induced \\
& - GPllb/llla inhibitor induced \\
\hline
\end{tabular}

\begin{abstract}
${ }^{1-2}$ Department of Internal Medicine and Critical Care, Virinchi Hospitals, Hyderabad, Telangana, India

Corresponding Author: Srinivas Samavedam, Department of Internal Medicine and Critical Care, Virinchi Hospitals, Hyderabad, Telangana, India, e-mail: srinivas3271@gmail.com

How to cite this article: Aluru N, Samavedam S. Thrombocytopenia in Intensive Care Unit. Indian J Crit Care Med 2019;23(Suppl 3):S185S188.

Source of support: Nil

Conflict of interest: None
\end{abstract}

- Enhanced consumption

- Pseudothrombocytopenia.

\section{Pseudothrombocytopenia}

This is a laboratory artifact usually caused by in vitro platelet agglutination in EDTA-anticoagulated blood. Platelet clumping occurs due to a naturally occurring autoantibody against an epitope on GPIIb/IIla. ${ }^{4}$ Platelet count is falsely low because the blood analyzer does not count the platelet clumps and platelet counts in citrate anticoagulated blood are usually normal, but not always. Pseudothrombocytopenia may also occur from in vitro adherence of platelets to leukocytes, known as satellitism, which can be identified by examination of the peripheral blood smear. It has been consistently observed in 1 in 1000 individuals and is not related to the presence or absence of the disease. Pseudothrombocytopenia has no major clinical implications except that most of the patients end up with unnecessary transfusion.

\section{Decreased Production and Increased Sequestration}

The extent to which these two mechanisms contribute to thrombocytopenia in the ICU is not known, and are unlikely to be dominant factors unless the patient has preexisting problem, with few exceptions. For example, acute alcohol intoxication is known to cause thrombocytopenia through decreased production, and in patients with sickle cell disease where splenic sequestration crisis is known to cause thrombocytopenia. In disease states with high proinflammatory activity like sepsis, erythropoiesis is blunted, but thrombopoiesis is stimulated, and hence a rare cause.

\section{Increased Destruction, Consumption, or Both}

Destruction and/or consumption of platelets, due to both immune and nonimmune mediated mechanisms, explains the most common cause of thrombocytopenia in the ICU. Probable mechanisms include thrombin-mediated platelet activation, development of antibodies, hemophagocytosis, complement activation, histone mediated platelet aggregation, ADAM ST 13 depletion. The classical clinical scenarios are patients with trauma and sepsis.

(0) The Author(s). 2019 Open Access This article is distributed under the terms of the Creative Commons Attribution 4.0 International License (https://creativecommons. org/licenses/by-nc/4.0/), which permits unrestricted use, distribution, and non-commercial reproduction in any medium, provided you give appropriate credit to the original author(s) and the source, provide a link to the Creative Commons license, and indicate if changes were made. The Creative Commons Public Domain Dedication waiver (http://creativecommons.org/publicdomain/zero/1.0/) applies to the data made available in this article, unless otherwise stated. 


\section{Sepsis}

Multiple mechanisms have been proposed to explain the thrombocytopenia of sepsis. The relative contribution of each potential mechanism may vary among patients and in the same patient over time. Enhanced-platelet consumption results from ongoing thrombin generation and increased adhesion of platelets to endothelial cells. ${ }^{4}$ Extensive endothelial activation characteristic of sepsis is associated with the release of large amounts of vWf multimers and reciprocally decreased amounts of the multimercleaving proteases, ADAMTS13. The exaggerated endothelial activation allows large numbers of platelets to be attached to the vascular endothelial cells, leading to thrombocytopenia. There is also increasing evidence for platelet interaction with white cells through the formation of plateletneutrophil aggregates and platelet-monocyte complexes in sepsis and other inflammatory conditions. A newly identified reason for thrombocytopenia in critical illness is the effect of extracellular histones. Animal studies have shown that rapid histone infusion causes rapid and profound thrombocytopenia, through platelet aggregation. This aggregatory effect is mediated by the activation of integrins and crosslinking of platelets and fibrinogen. The studies also show that high histone levels during ICU stay strongly predict the development of moderate to severe thrombocytopenia.

\section{Common Causes}

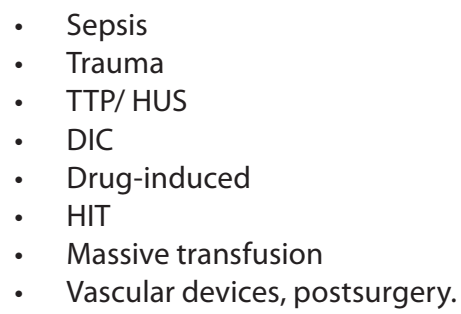

\section{Sepsis}

Sepsis accounts for up to $50 \%$ incidence of thrombocytopenia in critically ill and is most often multifactorial. Thrombocytopenia is known to independently modify the host immune response to sepsis. Hemophagocytosis is a frequent cause of unexplained thrombocytopenia in patients with severe sepsis syndrome.

\section{Trauma}

Trauma-induced coagulopathy together with hemodilution due to massive transfusion is a common cause. along with consumption, hyper fibrinolysis and systemic inflammation. ${ }^{4}$

\section{TTP/HUS}

TTP is described as a pentad of fever, thrombocytopenia, MAHA, renal dysfunction, and neurological impairment, often some of these features are not present and often may be confused with HUS, which is most commonly characterized by the triad of thrombocytopenia, MAHA, and renal dysfunction. ${ }^{5}$ These clinical similarities of DIC, TTP, and HUS are a major concern because they pose a risk of misdiagnosis, as clinicians are more likely to consider a diagnosis of DIC than of the rarer TTP or HUS, thus delaying potentially lifesaving treatment. ${ }^{6}$ Hemolytic anemia, negative Coombs test, elevated LDH levels, and organ dysfunction with thrombocytopenia are common to DIC, TTP, and HUS. The most important distinguishing factor between DIC and TTP/HUS is the coagulation profile, as patients with DIC have altered coagulation. ${ }^{7}$
Table 2: Drugs causing thrombocytopenia, associated with drugdependent antibodies

$$
\begin{aligned}
& \text { - Vancomycin } \\
& \text { - Trimethoprim-sulfamethoxazole } \\
& \text { - Piperacillin } \\
& \text { - Rifampin } \\
& \text { - Oxaliplatin } \\
& \hline
\end{aligned}
$$

\section{DIC}

DIC is relatively common, developing in $9-19 \%$ of ICU patients, usually as a result of sepsis. DIC may not only be viewed as a coagulation disorder, but also as a delayed symptom of emerging systemic vascular inflammatory disease and endothelial dysfunction. ${ }^{8}$ In the early 1990 s DIC was regarded merely as a terminal phase of MODS, but off late it has been recognized as a syndrome that requires precise diagnosis and treatment, ${ }^{9}$ and the ISTH has developed diagnostic criteria for diagnosis for DIC, which are now widely employed.

\section{Drug-induced Thrombocytopenia}

Many drugs cause thrombocytopenia (Table 2). Thrombocytopenia caused by drug-dependent antibodies occurs suddenly, is typically severe, is often misdiagnosed and therefore commonly recurs. ${ }^{10}$ Drug-induced nonimmune thrombocytopenia is a frequent cause, and with exception of HIT immune-mediated thrombocytopenia is less frequent. Laboratory tests for the detection of drug-dependent antiplatelet antibodies are helpful to support the diagnosis; however, these tests are performed only in specialized laboratories and are usually not available to guide acute management. The clinical relevance of laboratory testing for drug-induced antibodies is twofold. First, it allows objective confirmation of an adverse drug effect. Second, it is important for the individual patient so as to avoid future exposure to the specific drug.

\section{HIT}

It is one of the most important adverse drug events encountered by physicians. It is a life threatening immune mediated prothrombotic state, most commonly encountered with UFH, and to a lesser extent with $\mathrm{LMWH}$, associated with platelet-activating anti-PF4/ heparin antibodies. ${ }^{8,11}$ Incidence of HIT varies widely among studies because of the wide range of underlying comorbidities and coadministration of many drugs simultaneously in the critically ill. Timing of onset, the moderate nature of thrombocytopenia, and the common concurrence of thrombosis, are very important factors to differentiate HIT from other explanations for thrombocytopenia in the ICU patient. Several clinical scoring systems have been proposed for diagnosis of HIT, the most widely employed is the 4T score. Similarly, various laboratory investigations are also available for the diagnosis of HIT, but the gold standard for diagnosis at this point of time is the serotonin release assay.

\section{Significance of Thrombocytopenia Developing during ICU Admission}

Multiple studies have shown that the development of thrombocytopenia during an ICU admission is associated with increased length of stay, morbidity, and mortality. ${ }^{1}$ This association has been noted in both medical and surgical patients, in pediatric and adult patients, and is particularly apparent as a prognostic indicator in sepsis. ${ }^{12}$ Patients who developed thrombocytopenia 
had more episodes of major bleeding compared to those patients who did not develop thrombocytopenia and also received more blood product transfusions. Thrombocytopenia has also been associated with ARDS. ${ }^{3}$ Several experimental and clinical observations have demonstrated that platelets are integral to the barrier function of the alveolar capillary endothelium. Thrombocytopenia can lead to disruption of this barrier integrity and lead to pulmonary edema, characteristic of ARDS. Animal data show that during mechanical ventilation, platelets release chemotactic proteins, which promote leukocyte recruitment and proinflammatory mediators characteristic of ARDS.

Of late the relation between low platelets at admission and host response has been studied in septic patients, which has shown a positive correlation of low-platelet count with increased cytokines and endothelial-cell activation and impaired vascular integrity. Impaired leukocyte-adhesion and increased complementsignaling suggesting impaired host-response. Admission platelet counts do not differentiate between survivors and nonsurvivors, but rather a persistently low-platelet count and the duration of thrombocytopenia were crucial for prognosis.

\section{Approach to Thrombocytopenia in ICU}

- A detailed history and careful physical examination are keys to achieving the right diagnosis. Systematic and extensive investigations are required to establish the cause of thrombocytopenia in ICU.

- To understand the likely causes of patient's thrombocytopenia, it is essential to have a full picture of the prior medical history, comorbidities, and medications.

- Always obtain previous CBC report to look at, if the patient has unrecognized preexisting disorders, for example: CLD, ITP

- The illness that led to the ICU admission should be clearly understood, e.g. a patient admitted with UTI, may become progressively worse and may land up with septic shock, MODS, which itself is known to cause thrombocytopenia, or the HPS secondary to sepsis.

- With reference to the course of illness, always look at the $C B C$ obtained during early course of the disease.

- Always rule out laboratory artifacts.

- Look at the timing of platelet drop, for example: acute drop $>50 \%$ in less than 48 hours, after any intervention or procedure goes in favor of thrombocytopenia secondary to the intervention, i.e. thrombocytopenia developing after giving drug known to cause low platelets, like vancomycin or a patient developing low count after insertion of a vascular device like IABP, TPI.

- Similarly HIT is known to occur after 5 days of exposure to UFH, unless patient has been previously exposed to unfractionated heparin.

- Look for evidence of hemolysis/abnormalities in the coagulation profile, where in the diagnosis narrows down to DIC, MAHA, TTP.

- Any associated thrombosis narrows down the diagnosis. HIT is a classic example, DIC associated with malignancy is another presentation.

- If thrombocytopenia is a part of pancytopenia, bone marrow examination is required to r/o malignancy.

Every patient with thrombocytopenia in ICU need not be investigated unless:

- Platelet count $<1000,000$

- $30 \%$ drop in platelet count
- Rapid decline in platelet count

- Failure to increase after 5-7 days of appropriate therapy

- Decline in platelet count after initial recovery.

\section{Management}

Thrombocytopenia is usually a consequence of the underlying illness, specific management of the underlying disease should result in improvement of the platelet count and hence importance should be given to appropriately treating the condition. Spontaneous bleeding is the biggest fear a clinician faces while treating patient with thrombocytopenia. Life threatening bleeding is usually rare with a platelet count $>20,000$ and rarely requires transfusion, unless the patient is bleeding or is planned for invasive procedures/ surgery. Randomized trials of transfusion strategies to treat or prevent bleeding in thrombocytopenic ICU patients have not been conducted, and a conservative approach seems warranted. The challenge faced by the Intensivist is to prevent unnecessary transfusion and at the same time keeping in view the risk of massive bleeding if not transfused.

- The most commonly accepted platelet count threshold for transfusions are a level of $10 \times 10^{9} / \mathrm{L}$ for those without and $20-30 \times 10^{9} / \mathrm{L}$ for those with additional risk factors for bleeding, such as concomitant coagulopathy (DIC) or severe hepatic or renal dysfunction. ${ }^{13}$

- If platelet dysfunction is a possibility, a higher threshold of $50 \times$

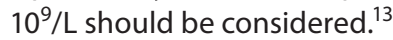

- If neurological complications like intracranial bleed may have occurred, a threshold of $100 \times 10^{9} / \mathrm{L}$ has been suggested. ${ }^{14}$

- In those patients who are not bleeding, platelet transfusions may be required if there is a need for surgical or radiological intervention.

- Thresholds for platelet counts have been set for many procedures which are often undertaken in the ICU with very poor evidence-base.

\section{Specific Therapy}

Certain conditions like HIT and TTP have specific therapy, which when timely initiated has shown a good outcomes.

HIT: Main management of thrombocytopenia in HIT is to stop UFH/ LMWH in any form and initiate the patient on alternate forms of anticoagulation like the Direct thrombin inhibitors and anti factor Xa agents.

TTP/HUS: Mortality in patients with TTP has shown to be close to $90 \%$ without plasma exchange, which is the main stay of management. Other therapies like steroids, cyclophosphamide, anti CD 20 agents, have also been tried, but with lesser efficacy as monotherapy compared to plasma exchange.

\section{Conclusion}

Thrombocytopenia in the ICU is common, especially among those with septic shock, and is often multifactorial. Careful clinical and laboratory investigations help to tailor the cause in most of the situations. In addition to evaluation for the cause of thrombocytopenia, the clinician must also guard against spontaneous bleeding, and treat active bleeding related to thrombocytopenia. Correction of the underlying cause will improve platelet counts in most of the cases, with a very few presentations which has specific therapies. 


\section{References}

1. Crowther MA, Cook DJ, Meade MO. Thrombocytopenia in medicalsurgical critically ill patients: prevalence, incidence, and risk factors. J Crit Care. 2005;20(4):348-353.

2. Thrombocytopenia in the critically ill: prevalence, incidence, risk factors, and clinical outcomes. Canadian Journal of Anesthesia/ Journal canadien d'anesthésie. July 2013;60(7):641-651.

3. Greinacher A, Selleng K. Thrombocytopenia in the intensive care unit patient. Hematology Am Soc Hematol Educ Program. 2010;2010:135143.

4. Andreas Greinacher, Sixten Selleng. How I evaluate and treat thrombocytopenia in the intensive care unit patient. Blood 2016 128:3032-3042; doi:https://doi.org/10.1182/blood-2016-09-693655

5. Drews RE. Critical issues in hematology: Anemia, thrombocytopenia, coagulopathy, and blood product transfusions in critically ill patients. Clin Chest Med 2003;24(4):607-622. DOI:10.1016/s02725231(03)00100n

6. James N. George, M.D. Thrombotic Thrombocytopenic Purpura. N Engl J Med 2006;354:1927-1935. DOI: 10.1056/NEJMcp053024

7. Barbara Ferrari, Alberto Maino, Luca A Lotta, Andrea Artoni, Silvia Pontiggia, Silvia M Trisolini, Alessandra Malato, Frits R Rosendaal, and Flora Peyvandi. Pregnancy complications in acquired thrombotic thrombocytopenic purpura: a case-control study. Orphanet J Rare Dis. 2014;9:193. doi: 10.1186/s13023-014-0193-6
8. Robert I Parker. Etiology and significance of thrombocytopenia in critically ill patients. Crit Care Clin. 2012 Jul;28(3):399-411, vi. doi: 10.1016/j.ccc.2012.04.007.

9. Satoshi Gando, Ferhat Meziani and Marcel Levi. What's new in the diagnostic criteria of disseminated intravascular coagulation? Intensive Care Med (2016) 42:1062-1064. DOI 10.1007/s00134-0164257-z

10. Aster RH, Bougie DW. Drug-induced immune thrombocytopenia. $\mathrm{N}$ Engl J Med. 2007 Aug 9;357(6):580-7.

11. Tsai, H.-M. (2007). Thrombotic Thrombocytopenic Purpura: A Thrombotic Disorder Caused by ADAMTS13 Deficiency. Hematology/ Oncology Clinics of North America, 21(4);609-632. doi:10.1016/j. hoc. 2007.06.003

12. Hui P, Cook DJ, Lim W, Fraser GA, Arnold DM. The frequency and clinical significance of thrombocytopenia complicating critical illness: a systematic review. Chest 2011;139:271-278.

13. Jean-Louis Vincent, Pedro Castro, Beverley J. Hunt, Achim Jörres, Manuel Praga, Jose Rojas-Suarez \& Eizo Watanabe. Thrombocytopenia in the ICU: disseminated intravascular coagulation and thrombotic microangiopathies - what intensivists need to know. Critical Care 2018;22:158

14. Afshar M, and Netzer G. (2013). Update in Critical Care for the Nephrologist: Transfusion in Non hemorrhaging Critically III Patients. Advances in Chronic Kidney Disease, 20(1);30-38. doi:10.1053/j. ackd.2012.10.007. 